\title{
PAX5 Haploinsufficiency Induces Immune Inhibitory-Related Molecules Expressions of CD8 + T Cells in the Tumor Microenvironment
}

mi liang

Tongji Hospital of Tongji Medical College of Huazhong University of Science and Technology duanhao gong

Tongji Hospital of Tongji Medical College of Huazhong University of Science and Technology

lei wang

Tongji Hospital of Tongji Medical College of Huazhong University of Science and Technology xue liang

Tongji Hospital of Tongji Medical College of Huazhong University of Science and Technology jiao meng

Tongji Hospital of Tongji Medical College of Huazhong University of Science and Technology wei huang ( 2896607051@qq.com)

Hua Zhong University of Science and Technology

jianfeng zhou

Tongji Hospital of Tongji Medical College of Huazhong University of Science and Technology

\section{Research}

Keywords: PAX5, Haploinsufficiency, immune inhibitory-related molecules

Posted Date: March 31st, 2021

DOl: https://doi.org/10.21203/rs.3.rs-355607/v1

License: (9) This work is licensed under a Creative Commons Attribution 4.0 International License. Read Full License 


\section{Abstract}

Loss of function of PAX5 plays an important role in PAX5 mutation tumor, and PAX5 haploinsufficiency promoting tumorigenesis is related to immune escape. But the mechanisms of PAX5 mutations inducing tumor immune escape have not been clarified. We estimated the proportions of 22 immune cell types and the expressions of immune inhibitory-related molecules based on gene expression profiles (GEPs) from Bacute lymphoblastic leukemia(B-ALL) with PAX5 mutations by CIBERSORT, an established algorithm. We constructed the PAX5 haplodeletion A20 cell lines, built allografted A20 tumor models and evaluated the effect of PAX5 haplodeletion on immune inhibitory-related molecules in the tumor microenvironment (TME). Our results indicated the percentages of T cells in bone marrow of B-ALL with PAX5 mutations were not statistically different from that in bone marrow of B-ALL without PAX5 mutations, except for T follicular helper (Tfh) cells. But a variety of up-regulated immune inhibitory-related molecules in bone marrow of B- ALL with PAX5 mutations were identified. By different approaches, we found that several immune inhibitory-related molecules of CD8+ T cells in TME of PAX5 haplodeletion clones such as TIM3, NR4A1 and BATF, were increased significantly compared with that of PAX5 wild type control. The IFN- $\triangle$ of CD8+ T cells in TME of PAX5 haplodeletion tumors was decreased significantly compared with that of PAX5 wild type control. Our study showed that PAX5 haploinsufficiency induced high expressions of TIM3, NR4A1 and BATF in the TME and was involved in CD8+ T cells dysfunction or exhaustion.

\section{Introduction}

The paired box domain gene 5 (PAX5) is one of the key genes encoding a paired box domain transcription factor essential for B-cell differentiation. PAX5 activates central genes for B-cell lineage differentiation and inhibits genes necessary for commitment in other lineages [1]. PAX5 alterations have been detected frequently in both childhood [2] and adult [3, 4] B- acute lymphoblastic leukemia (B-ALL). PAX5 alterations have also been detected in Hodgkin lymphoma [5] and non-Hodgkin lymphoma [6, 7]. Differential PAX5 levels were related to prognosis in mantle cell lymphoma independent of CCND1[8]. Reduced PAX5 levels in CD19 + MCL cells influenced their increased tumor infiltration and progression [8]. The recent clinical data from childhood B-ALL showed that PAX5 deletion was an independent risk factor for disease-free survival in B-ALL children without reproducible chromosomal abnormalities [9]. So PAX5 mutations have a key role in leukemogenesis and leukemia relapse.

PAX5 sequence mutations are common PAX5 alterations of B cells tumors $[2,3,10,11]$. Of the PAX5 sequence mutations, $56 \%$ were heterozygous mutations [11]. The B-ALL PAX5 alterations is also remarkable for the near-universal inactivation of the wild-type PAX5 allele, by deletion, acquired copyneutral loss of heterozygosity that duplicates the mutant allele or acquisition of a second PAX5 sequence mutation that causes loss of function [11]. So heterozygous mutations and rearrangements, like homozygous mutations of PAX5, also result in dysfunction of PAX5. The previous study showed that dysfunction of PAX5 plays a key role in the PAX5 mutation tumor. PAX5 haploinsufficiency (not PAX5-/-) synergized with STAT5 activation to initiate acute lymphoblastic leukemia [12]. Another study showed that PAX5 haploinsufficiency (not PAX5-/-) exploited Sca1-BCR-ABLp190 susceptibility to confer the shift 
essential for pB-ALL [13]. So, we chose PAX5 haploinsufficiency to to investigate the role of PAX5 alterations in the pathophysiology of hematologic tumors.

Tumor immune escape takes part in leukemiogenesis and leukemia relapse. The presence of CD $8+T$ cells within the tumor microenvironment (TME) is the most predictive biomarkers for response to cancer immunotherapy. Currently, it is believed that there are two modes of tumor immune escape in TME: T-cell inflamed tumor and non-T-cell inflamed tumor. For T-cell inflamed tumors, there are CD $8+T$ cells in TME accompanied by the up-regulation of immune inhibitory mechanisms, which inhibit the anti-tumor effect of CD8 $+T$ cells [14]. Up-regulation of immune inhibitory-related molecules in CD8 $+T$ cells played an important part in T cell dysfunction or exhaustion within the TME [15].

In our previous studies, PAX5 haploinsufficiency promoting tumorigenesis may be related to immune escape [16]. Recent studies have found that PAX5 mutations were involved in B-ALL relapse after anti CD19 chimeric antigen receptor (CAR) T -cell treatment $[17,18]$. There was a paucity of research on the mechanisms of PAX5 mutations causing immunologic escape. To clarify the mechanisms of PAX5 mutations causing immunologic escape, we firstly analyzed proportions of 22 immune cell types and immune inhibitory-related molecules in bone marrow of B - ALL with PAX5 mutations using the Cell-type Identification by Estimating Relative Subsets of RNA Transcripts (CIBERSORT) algorithm and validated immune inhibitory-related molecules in TME using the PAX5 haplodeletion tumor mice models. Our study showed PAX5 haploinsufficiency induced up-regulation of immune inhibitory -related molecules in CD8 + $T$ cells in TME and was involved in CD8 $+T$ cells dysfunction or exhaustion.

\section{Materials And Methods}

\section{Data collection}

The mRNA profiling and clinical data (age, gender, WBC, and CNS status) were obtained from the database of Gene Expression Omnibus (GEO http://www.ncbi.nlm.nih.gov/geo, accession number: GSE11877). Cytogenetic abnormalities of 134 patients were obtained from Mullighan[10], which excluded the cases with known very high-riskALL subtypes (i.e., BCR-ABL1-positive ALL, hypodiploid ALL, ETV6RUNX1, and ALL in infants).These patients included 70 cases with PAX5 mutations and 64cases without PAX5 mutations.

\section{Assessment of immune cell types}

As a deconvolution algorithm, CIBERSORT can estimate the cell composition of complex tissues based on normalized GEPs. We used CIBERSORT (http://cibersort.stanford.edu/) to estimate the relative fractions of immune cell types in bone marrow of B-ALL. These immune cell types in bone marrow included Tcells(Tfh cells, resting memory CD4 + T cells, activated memory CD4 + T cells, $y \delta ~ T$ cells, CD8 + T cells, Tregs, naïve CD4 + T cells), B cells (memory B cells, naïve B cells, plasma cells), NK cells (activated and resting NK cells), and myeloid subsets (M0 macrophages, M1 macrophages, M2 
macrophages, activated and resting mast cells, activated and resting dendritic cells, neutrophils, monocytes and eosinophils) [19].

Cell culture

The BALB/c B cell lymphoma line A20 was purchased from China Center for Type Culture Collection (CCTCC, Wuhan, China) and HEK293T was purchased from CCTCC (Wuhan, China). A20 cells were cultured in RPMI 1640 medium containing 5-10\% fetal calf serum (FCS, Invitrogen, United States), HEK293T cells were cultured in DMEM supplemented with $10 \%$ dialyzed fetal bovine serum.

Construction and identification of PAX5+/-A20 cell clone

Construction and identification of PAX5+/-A20 cell clone were carried out in a way that we previously established [44].

The vector pSpCas9(BB) -2A-GFP (PX458)(Plasmid \#48138)was bought from Addgene (Massachusetts, United States). The oligo DNA targeting the PAX5 exon3 locus was designed on the MIT online software ZhangFeng lab: http://crispr.mit.edu/. The primers used for knockout were synthesized in Invitrogen company (see the Supplementary Appendix Table 1). We formed dsDNA using Precut sgRNA Cloning kit and pSDgRNA Plasmid construction Kit (Biomics Biotechnologies Co., Ltd, Jiangsu, China) according to the instruction. Next, Cas9/sgRNA plasmids targeting PAX5 were constructed, amplified, purified with EndoFree Plasmid Maxi Kit (QIAGEN, Germany), and were validated by sequencing. The Cas9/sgRNA plasmids were electrotransfected into HEK293T cells. The T7 Endonuclease I assay was applied to survey the efficiency of NHEJ-mediated mutations in the endogenous PAX5 gene. The most efficient Cas9/sgRNA plasmid was chosen for follow-up research.

Table 1

Clinical features of the patient cohorts

\begin{tabular}{|c|c|c|c|}
\hline Clinical Features & $\operatorname{PAX} 5+(n=70)$ & PAX5- $(n=64)$ & $p$ value \\
\hline Gender & & & $\nabla 0.05$ \\
\hline Male & $46(66 \%)$ & $43(67 \%)$ & \\
\hline Female & $24(34 \%)$ & $21(33 \%)$ & \\
\hline Age (months) & & & $\varangle 0.05$ \\
\hline Median & 146 & 171 & \\
\hline Range & $17-249$ & $12-222$ & \\
\hline WBC (1000/ul) & $105 \pm 118$ & $103 \pm 145$ & $\otimes 0.05$ \\
\hline CNS disease & $16(23 \%)$ & $12(19 \%)$ & $\varangle 0.05$ \\
\hline
\end{tabular}


$3 \times 10^{5}$ A20 cells were collected and suspended with the matched solution supplemented with $5 \mu \mathrm{g}$ CRISPR/CAS9 plasmid. Electrotransfection was performed on LONZA 4D Nucleofector System (Lonza, Switzerland). Cells were cultured for $48 \mathrm{~h}$ and then sorted with Beckman MoFlo XDP (Beckman Coulter, Inc., American), aiming to select cells with high GFPs expression. The sorted cells were seeded in 96-well plates in the manner of a single cell. 2-3 weeks' later, cells were collected for identification. Genomic DNA was isolated using the DNA Isolation Kit (BioTeke Corporation, Beijing, China), and PAX5 gene was amplified with PCR for mutation analysis. The PCR primers were synthesized by Sangon Biotech (Shanghai, China) (see the Supplementary Appendix Table 2). The PCR product was reclaimed for sequence determination. The sequencing results were compared with the published PAX 5 gene sequence to determine the presence of PAX5+/- variants.

Cells were lysed with RIPA lysis buffer (Beyotime, Shanghai, China) added with a protease inhibitor cocktail (Roche, Switzerland). The protein concentration was measured by the cinchoninic acid protein assay (Thermo Scientific, United States). $30 \mu \mathrm{g}$ of total lysate was subjected to SDS-PAGE and then transferred to nitrocellulose membranes (Bio-Rad Laboratories, United States). The membranes were incubated with the antibodies against PAX5 and B-actin purchased from Abcom Biotechnology (Abcam, CA, United States), and they were blotted with corresponding HRPlinked secondary antibodies. The proteins were measured using an enhanced ECL system (Pierce, United States). Quantification was performed with ImageJ (https://imagej.nih.gov/) and each sample's ratio relative to the loading control $\beta$ actin was calculated.

Structuring allografted tumor models

All methods were fulfilled based on the Guidelines for the Care and Use of Laboratory Animals of the Council of Science and Technology of China. All test protocols were approved by the Animal Care and Use Committee of Tongji Hospital. Six-weeks old female BALB/c mice were bought from the Animal Center of Tongji Medical College. All mice were fed in individually ventilated, pathogen-free cages where they had access to food and water optionally. Tumor size, weight, activity, and skin changes were monitored carefully throughout the experiments. No animals showed signs of illness following tumor formation and none died due to the experimental procedure.

Forty mice were divided at random into four groups: PAX5+/- mice (MUT1, $n=10 ;$ MUT2, $n=10$; MUT3, $n$ $=10)$ and PAX5 wild type mice $(W T, n=10) .5 \times 10^{6}$ cells in $0.15 \mathrm{ml}$ PBS were subcutaneously injected into the belly of BALB/c mice. The growth of the allografted tumors was observed for two weeks. After two weeks, the mice were fully anesthetized using Ketalar-Dormicum and sacrificed with CO2. We observed whether allografted tumors formed and tumor weight was measured. And then, Tumor specimens were used for the next research.

Sorting of $\mathrm{CD} 8+\mathrm{T}$ cells

Tumors were cut into small fragments and digested, and single-cell suspensions were prepared. CD8 + T cells were sorted using anti-mouse CD8a MicroBeads (Miltenyi Biotec Inc, United States) according to the 
manufacturer's instructions. The purity of CD8 + T cells was typically greater than $90 \%$ by fluorescence activating cell sorter.

\section{Quantitative RT-PCR}

Total RNA was extracted with TRIzol reagent (Invitrogen, United States), and was reverse transcribed into cDNA using the Hieff cDNA Synthesis Super Mix (Yeasen Biotech, Shanghai, China). All primers were shown (see the Supplementary Appendix Table 2). The Applied Bio-systems StepOneTM RT-PCR machine was used to carry out quantitative PCR with the Hieff qPCR SYBR Green Master Mix (Yeasen Biotech, Shanghai, China). All reactions were repeated at least in triplicate. We used endogenous GAPDH to normalize the sample with the comparative threshold cycle (Ct) method for relative quantification.

Flow cytometry

To quantify levels of immune inhibitory molecules of $C D 8+T$ cells in tumor specimens, single cell suspensions were surface or intracellular stained with the following fluorescent antibodies:CD45-APCcy7, CD3-APC, CD8-PerCP, TIM3-PE-Cy7, BATF-PE, NR4A1-FITC, IFN-X-BV510(BD Biosciences,Germany). For BATF,NR4A1 staining, cells were fixed and permeabilized with the Transcription Factor staining buffer

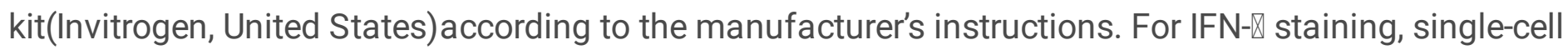

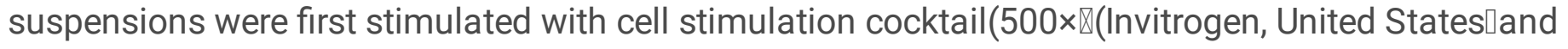
inhibited with protein transport inhibitor(500×凶(Invitrogen, United States), and then fixed and permeabilized with Cytofix/Cytoperm Fixation/Permeabilization Solution Kit(BD Biosciences,Germany).Stained samples were collected and analyzed using a FACScan flow cytometer (BD Biosciences,Germany). Finally, all the data were analyzed by the FlowJo software ( 10 ,United States).

\section{Statistical analysis}

Statistical analyses of the mRNA profiling from B-ALL were conducted using the "CIBERSORT.R" package and Bioconductor (https://www.bioconductor.org/). Each dataset was processed by a weighted average method to compare the differences in the composition of immune cell types and the mRNA levels of immune inhibitory -related molecules between cases with PAX5 mutations and cases without PAX5 mutations. All analyses were conducted using R Studio (version 4.0.3).

The results from mice were expressed as the mean and standard deviation (SD) of at least three independent experiments and three repetitions. Statistical significance was estimated by Student's t-tests or one-way analysis of variance (ANOVA) followed by Dunnett's test. The statistical analyses were performed using the Graphpad Prism (version 8.0) software. A p-value of $<0.05$ was significant.

\section{Results}

Clinical Features of the patient cohorts 
134 cases were divided into two groups: 70 cases with PAX5 mutations and 64 cases without PAX5 mutations. There was no difference between the two groups in gender, age, white blood count (WBC) and central nervous system (CNS) leukemia (Table 1).

The distribution of immune cell subtypes in bone marrow of B-ALL.

We first explored the distribution of immune cell subtypes in bone marrow of B-ALL by using the CIBERSORT algorithm. The gene expression profiles (GEPs) of 134 cases of B-ALL were analyzed using CIBERSORT, the total proportion of immune cell subtypes was shown in Fig. 1A.

There was a statistically significant difference in the percentages of $T$ follicular helper (Tfh) cells and naïve CD $4+T$ cells in bone marrow between B-ALL with PAX5 mutations and that without PAX5 mutations $(p<0.05)$. The percentages of macrophages showed statistical differences between B-ALL without PAX5 mutations and with PAX5 mutations $(p<0.05)$. The percentage of CD8 $+T$ cells in bone marrow of B-ALL with PAX5 mutations was lower than that in bone marrow of B-ALL without PAX5 mutations, but there was no statistically significant difference between two groups. There were statistically significant differences between two groups in levels of immune inhibitory molecules, such as PD-1, CTLA-4, LAG-3, mTOR, TIM3, BATF, NR4A1, TIGIT, TCF-1, NFAT, CD160, EOMES, IL-10 ( $p<0.001)$ (Fig. 1B). The levels of these molecules in bone marrow B-ALL with PAX5 mutations were higher than that in bone marrow of B-ALL without PAX5 mutations.

Identification of PAX5+/- A20 cells

Genome editing tools have been widely used to modify genes in model systems including animal and human cells. We designed a gRNA that directed exon3 of PAX5, and then we inverted it into the cas 9 and GFP expressing vector. After the editing and subsequent screening process, we randomly chose the clones and checked out mutated clones by DNA sequencing. Sequencing results displayed that mutation clones had haplodeletion mutation in exon3 of PAX5 by comparison of DNA sequences of mutation clone with that of wild type control (Fig. 2A). Quantitative RT-PCR analysis displayed levels of mRNA in PAX5 mutation clones were significantly lower than that in wild-type control $(p<0.05)$ (Fig. 2B). Western blot showed levels of protein in PAX5 mutation clones were lower than that in wild-type control (Fig. 2C). The mRNA levels of CD79a (PAX5 downstream target gene) were significantly lower than that in wild-type control $(p<0.05)$ (Fig. 2D).

Effect of PAX5 haplodeletion on tumor

The tumor weights were measured to estimate the effect of PAX5 haplodeletion on tumor growth. The tumors from PAX5 haplodeletion clones grew significantly faster than tumors of PAX5 wild type control (Fig. 3)

Evaluating immune inhibitory -related molecules expression of CD8 $+\mathrm{T}$ cells in the TME 
To figure out the immune inhibitory-related molecules of CD8 + T cells in TME, we sorted out the CD $8+T$ cells, and detected mRNA of immune inhibitory-related molecules in CD8 + T cells. The results showed that the mRNA levels of TIM3, BATF, NR4A1 in PAX5 haplodeletion tumors were higher than that in wildtype tumors $(p<0.05)$ (Fig. 4A). We detected the protein levels of TIM3, BATF, NR4A1 of CD8 + T cells in PAX5 haplodeletion tumors by flow cytometry. The protein levels of TIM3, BATF, NR4A1 of CD8 + T cells in PAX5 haplodeletion tumors were higher than that in wild-type tumors $(p<0.05)$ (Fig. 4B).

To assess $C D 8+T$ cells function, we analyzed the IFN- $\triangle$ levels of $C D 8+T$ cells in TME. The protein levels of IFN- $\triangle$ in $C D 8+T$ cells of PAX 5 haplodeletion tumors were lower than that of wild-type tumors $(p<0.05)$

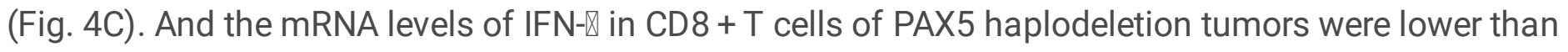
that of wild-type tumors $(p<0.05)$ (Fig.C). The results hinted dysfunction of CD8 + T cells in PAX5 haplodeletion tumors.

\section{Discussion}

The immune system has the greatest potential for the specific elimination of tumors, that can hold back cancer relapse. However, tumors acquire genetic mutations, lead to activation of immunosuppressive signaling pathways, and go through epigenetic changes that lead to immunologic escape. This immunologic escape reveals as an ability to avoid immune recognition or to avoid immune attack. Dysfunctional immune state in TME is a hallmark of cancer [20]. CD8 + T cells are the major anticancer effector cells in TME. For T cell-inflamed tumor, CD8 + T cells present in TME but run into a dysfunctional state termed T-cell exhaustion because of the up-regulation of immune inhibitory molecules or recruitment of regulatory T cells (Tregs) [21]. It is the current orientation of oncology therapy that searching immune inhibitory related molecules as targets for precise immunotherapy. The immunotherapy of anti-CTLA-4 and anti-PD-1 antibodies is the successful example of anti-cancer immunotherapy [22].

The PAX5 deletion was an independent risk for leukemia relapse in B- ALL children without reproducible chromosomal abnormalities (23). PAX5 mutations were involved in B-ALL relapse after anti CD19 CAR T cell treatment $[17,18]$. It remains unclear how PAX5 mutations are involved in immune escape. In our study, we applied the GEPs to describe the difference of immune cell subtypes in bone marrow between B-ALL with PAX5 mutations and B-ALL without PAX5 mutations. Only the percentages of Tfh cells, naïve CD4 $+T$ cells and macrophages was statistically significant in bone marrow between B-ALL with PAX5 mutations and B-ALL without PAX5 mutations. The percentage of Tfh cells in bone marrow of B-ALL with PAX5 mutations was significantly lower than that in bone marrow of B-ALL without PAX5 mutations. Some studies suggested that tumor infiltrating Tfh cells predicted improved disease outcome among breast carcinoma patients [24], but some people thought that Tfh cells was a double-edged sword in colorectal cancer [25]. The role of naïve CD4 + T cells and macrophages was also not explicit. As the key effector cells in TME, CD8 + T cells in bone marrow of B-ALL with PAX5 mutations was lower than that in bone marrow of B-ALL without PAX5 mutations, but there was no statistically significant difference 
between two groups. So, the distribution of immune cell subtypes could not explain all how PAX5 mutations were involved in tumor immune escape.

CD8 $+T$ cells are key mediators of antitumor function and are proven critical for cancer immunotherapy. $T$ cell dysfunction or exhaustion was considered one of the important mechanisms of tumor immune escape [26].

Dysfunctional CD8 + T cells are characterized by a loss of effector functions, such as attenuated

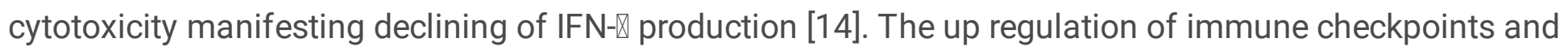
changes in transcriptional and metabolic molecules have been described as hallmarks of $T$ cell dysfunction [15]. We analyzed GEPs from bone marrow of B-ALL to identify the difference in these immune inhibitory-related molecules between B-ALL with PAX5 mutations and B-ALL without PAX5 mutations. We found there were statistical differences in some immune inhibitory-related molecules suchs as PD-1, CTLA-4, LAG-3, mTOR, TIM3, BATF, NR4A1, TIGIT, TCF-1, NFAT, CD160, EOMES, IL-10. By tumor models with PAX5 haplodeletion, we found the levels of TIM3, BATF, NR4A1 of CD8 + T cells were higher in TME of PAX5 haplodeletion tumors than that in TME of PAX5 wild-type tumors. And in TME of

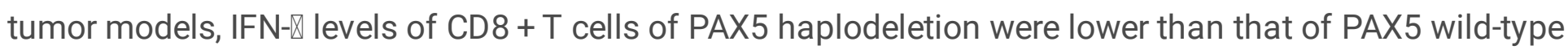
tumors, which indicated dysfunction or exhaustion of CD8 + T cells in PAX5 haplodeletion tumors.

TIM3 belonged to the Ig superfamily, and it could be detected on various immune cells. It was approved that TIM3 was a key regulator of the exhausted antigen specific CD8 + T cells that arose in both humans and mice during chronic viral infections, such as HIV, hepatitis $C$ virus (HCV), hepatitis $B[27,28,29]$. The research results of various tumors gave evidence that TIM3 was involved in T cell dysfunction or exhaustion [26]. TIM3 expression represented dysfunctional tumor infiltrating CD8 $+\mathrm{T}$ cells in renal cell carcinoma [30, 15]. TIM3 expression was related to poor prognosis of tumors [31, 32, 33]. Anti-TIM3 antibody promoted T cell IFN- $₫$ mediated anti-tumor immunity and suppresses tumors [34]. Many antiTIM3 clinical trials are ongoing and TIM3 might be one of promising therapeutic targets after PD1[35, 36, 37].

NR4A1 and BATF were transcription factors identified as exhaustion-related genes, which potentially played an important regulatory role in tumor-reactive $\mathrm{CD} 8+\mathrm{T}$ cells $[38,39]$. The knockdown of BATF using shRNA-mediated gene-silencing enhanced T cell function [40] and NR4A1 deletion enhanced immunity against tumor [23]. The function of BATF and NR4A1 was associated with immune checkpoints. PD-1 induced T cell dysfunction by upregulating BATF [40]. NR4A1 deficiency resulted in down-regulation of the inhibitory receptors PD-1 and TIM3[41]. The present studies suggested that transcription factors NR4A1 and BATF were involved in immune checkpoint-mediated T cell dysfunction or exhaustion.

The current studies found that the immune checkpoints-mediated T cell dysfunction or exhaustion was involved not only in tumor recurrence but also in failure of CAR T cells [41, 42]. In clinical trials of CAR T cells in hematologic malignancy, there was a case report that CAR T cells treatment failure were related to CAR T cell exhaustion caused by TIM3 and PD-1 signaling pathways [43]. 
In summary, our findings demonstrated that PAX5 haploinsufficiency induced high expression of TIM3, NR4A1 and BATF in the TME, and was involved in CD8 + T cells dysfunction or exhaustion.

\section{Declarations}

\section{Competing interests}

The authors declare that they have no conflicts of interest.

\section{Authors' contributions}

In this study, Mi Liang, Wei Huang and Jianfeng Zhou designed and supervised the study and experiments, analyzed the data, and co-wrote the manuscript. Duanhao Gong, Lei Wang, Xue Liang and Jiao Meng developed the methodologies, performed the experiments, analyzed the data. All authors read and approved the final manuscript.

\section{Funding}

This work was supported by a Grant from the National Natural Science Foundation of China (No. 81770164).

\section{References}

1. Medvedovic J, Ebert A, Tagoh H, Busslinger M. PAX5: a master regulator of B cell development and leukemogenesis. Adv Immunol. 2011; 111:179-206.

2. Mullighan CG, Goorha S, Radtke I, Miller CB, Coustan-Smith E, Dalton JD, Girtman K, Mathew S, Ma J, Pounds SB, Su X, Pui CH, Relling MV, Evans WE, Shurtleff SA, Downing JR. Genomewide analysis of genetic alterations in acute lymphoblastic leukaemia. Nature. 2007; 446:758-764.

3. Iacobucci I, Lonetti A, Paoloni F, Papayannidis C, Ferrari A, Storlazzi CT, Vignetti M, Cilloni D, Messa F, Guadagnuolo V, Paolini S, Elia L, Messina M, Vitale A, Meloni G, Soverini S, Pane F, Baccarani M, Foà $R$, Martinelli G. The PAX5 gene is frequently rearranged in BCR-ABL1-positive acute lymphoblastic leukemia but is not associated with outcome a report on behalf of the GIMEMA acute leukemia working party. Haematologica. 2010; 95:1683-1690.

4. Kim M, Choi JE, She CJ, Hwang SM, Shin HY, Ahn HS, Yoon SS, Kim BK, Park MH, Lee DS. PAX5 deletion is common and concurrently occurs with CDKN2A deletion in B-lineage acute lymphoblastic leukemia. Blood Cells Mol Dis. 2011; 47:62-66.

5. Liso A, Capello D, Marafioti T, Tiacci E, Cerri M, Distler V, Paulli M, Carbone A, Delsol G, Campo E, Pileri S, Pasqualucci L, Gaidano G, Falini B. Aberrant somatic hypermutation in tumor cells of nodularlymphocyte- predominant and classic Hodgkin lymphoma. Blood. 2006; 108:1013-1020.

6. Gaidano G, Pasqualucci L, Capello D, Berra E, Deambrogi C, Rossi D, Maria Larocca L, Gloghini A, Carbone A, Dalla-Favera R. Aberrant somatic hypermutation in multiple subtypes of AIDS-associated 
nonHodgkin lymphoma. Blood. 2003; 102:1833-1841.

7. Montesinos-Rongen M, Van Roost D, Schaller C, Wiestler OD, Deckert M. Primary diffuse large B-cell lymphomas of the central nervous system are targeted by aberrant somatic hypermutation. Blood. 2004; 103:1869-1875.

8. Teo AE, Chen Z, Miranda RN, McDonnell T, Medeiros LJ, McCarty N. Differential PAX5 levels promote malignant B-cell infiltration, progression and drug resistance, and predict a poor prognosis in $\mathrm{MCL}$ patients independent of CCND1. Leukemia.2016; 30:580-593.

9. Liu, XM, Zhang, L, Ruan, M, Liu, TF, Zhang, JY, Liu F, Qi BQ, Chen XJ, Wang SC, Yang WY, Guo Y, Zou Y, Chen YM, Zhu XF. Significance of PAX5 deletion in childhood B-lineage acute lymphoblastic leukemia without reproducible chromosomal abnormalities. Zhongguo Dang Dai Er Ke Za Zhi. 2016; 18:287-291.

10. Mullighan CG, Su X, Zhang J, Radtke I, Phillips LA, Miller CB,Ma J, Liu W, Cheng C, Schulman BA, Harvey RC, Chen IM, Clifford RJ, Carroll WL, Reaman G, Bowman WP, Devidas M, Gerhard DS, Yang WJ, Relling MV, Shurtleff SA, Campana D, Borowitz MJ, Pui CH, Smith M, Hunger SP, Willman CL, Downing JR. Deletion of IKZF1 and prognosis in acute lymphoblasticleukemia. N Engl J Med. 2009;360(5):470-80.

11. Gu Z, Churchman ML, Roberts KG, Moore I, Zhou X, Nakitandwe J, Hagiwara K, Pelletier S, Gingras S, Berns H, Payne-Turner D, Hill A, lacobucci I, Shi L, Pounds S, Cheng C, Pei D, Qu C, Newman S, Devidas M, Dai Y, Reshmi SC, Gastier-Foster J, Raetz EA, Borowitz MJ, Wood BL, Carroll WL, ZweidlerMcKay PA, Rabin KR, Mattano LA, Maloney KW, Rambaldi A, Spinelli O, Radich JP, Minden MD, Rowe JM, Luger S, Litzow MR, Tallman MS, Racevskis J, Zhang Y, Bhatia R, Kohlschmidt J, Mrózek K, Bloomfield CD, Stock W, Kornblau S, Kantarjian HM, Konopleva M, Evans WE, Jeha S, Pui CH, Yang J, Paietta E, Downing JR, Relling MV, Zhang J, Loh ML, Hunger SP, Mullighan CG. PAX5-driven subtypes of B-progenitor acute lymphoblastic leukemia. Nat Genet. 2019; 51(2):296-307.

12. Heltemes-Harris LM, Willette MJ, Ramsey LB, Qiu YH, Neeley ES, Zhang N, Thomas DA, Koeuth T, Baechler EC, Kornblau SM, Farrar MA. Ebf1 or Pax5 haploinsufficiency synergizes with STAT5 activation to initiate acute lymphoblastic leukemia. J Exp Med. 2011; 208(6):1135-49.

13. Martín-Lorenzo A, Auer F, Chan LN, García-Ramírez I, IGonzález-Herrero I, GRodríguez-Hernández G, Bartenhagen C, Dugas M, Gombert M, Ginzel S, Blanco O, Orfao A, Alonso-López D, Rivas JDL, GarcíaCenador MB, García-Criado FJ, Müschen M, Sánchez-García I, Borkhardt A, Vicente-Dueñas C and Hauer J. Loss of Pax5 Exploits Sca1-BCR-ABLp190 Susceptibility to Confer the Metabolic Shift Essential for pB-ALL. Cancer Res. 2018; 78(10):2669-2679.

14. Trujillo, JA, Sweis, RF, Bao, R, Luke, JJ. T Cell-Inflamed versus Non-T Cell-Inflamed Tumors: A Conceptual Framework for Cancer Immunotherapy Drug Development and Combination Therapy Selection. Cancer Immunol Res. 2018; 6:990-1000.

15. Zhang Z, Liu S, Zhang B, Qiao L, Zhang Y, Zhang Y. T Cell Dysfunction and Exhaustion in Cancer. Front Cell Dev Biol. 2020;8:17. 
16. Gu J, Li T, Zhao L, Liang X, Fu X, Wang J, Shang Z, Huang W, Zhou JF. Identification of Significant Pathways Induced by PAX5 Haploinsufficiency Based on Protein-Protein Interaction Networks and Cluster Analysis in Raji Cell Line.Biomed Res Int. 2017; 5326370.

17. Jacoby E, Nguyen SM, Fountaine TJ, Welp K, Gryder B, Qin H, Yang Y, Chien CD, Seif AE, Lei H, Song YK, Khan J, Lee DW, Mackall CL, Gardner RA, Jensen MC, Shern JF, Fry TJ. CD19 CAR immune pressure induces B-precursor acute lymphoblastic leukaemia lineage switch exposing inherent leukaemic plasticity. Nat Commun. 2016;7:12320.

18. Li X, Chen W.Mechanisms of failure of chimeric antigen receptor T-cell therapy.Curr Opin Hematol. 2019;26(6):427-433.

19. Ali HR, Chlon L, Pharoah PD, Markowetz F, Caldas C, Patterns of immune in-filtration in breast cancer and their clinical implications: a gene-expression-Basedretrospective study. PLoS Med. 2016;13 (12) e1002194.

20. Hanahan D, Weinberg RA.Hallmarks of cancer: the next generation.Cell.2011; 144: 646-74.

21. Spranger S. Mechanisms of tumor escape in the context of the T-cell-inflamed and the non-T-cellinflamed tumor microenvironment. Int Immunol. 2016; 28:383-91.

22. Rotte A. Combination of CTLA-4 and PD-1 blockers for treatment of cancer.J Exp Clin Cancer Res. 2019; 38: 255.

23. Liu X, Wang Y, Lu H, Li J, Yan X, Xiao M, Hao J, Alekseev A, Khong H, Chen TH, Huang R, Wu J, Zhao Q, Wu Q, Xu SL, Wang XH, Jin W, Yu SC, Wang Y, Wei L, Wang A, Zhong B, Ni L, Liu XL, Nurieva R, Ye L, Tian Q, Bian XW, Dong C. Genome-wide analysis identifies NR4A1 as a key mediator of T cell dysfunction. Nature. 2019;567(7749): 525-9.

24. Gu-Trantien C, Loi S, Garaud S, Equeter C, Libin M, de Wind A, Ravoet M, Le Buanec H, Sibille C, Manfouo-Foutsop G, Veys I, Haibe-Kains B, Singhal SK, Michiels S, Rothé F, Salgado R, Duvillier H, Ignatiadis M, Desmedt C, Bron D, Larsimont D, Piccart M, Sotiriou C, Willard-Gallo K. CD4+ follicular helper T cell infiltration predicts breast cancer survival. J Clin Invest. 2013; 123(7):2873-92.

25. Hetta HF, Elkady A, Yahia R, Meshall AK, Saad MM, Mekky MA, Al-Kadmy IMS. T follicular helper and $T$ follicular regulatory cells in colorectal cancer: A complex interplay. J Immunol Methods.2020;480:112753.

26. Wherry EJ. T cell exhaustion.Nat Immunol. 2011;12(6):492-9.

27. Amancha PK, Hong JJ, Ansari AA, Villinger F. Up-regulation of TIM3 on T cells during acute simian immunodeficiency virus infection and on antigen specific responders. AIDS. 2019;29:531-536.

28. Golden-Mason L, Waasdorp Hurtado CE, Cheng L, Rosen HR. Hepatitis C viral infection is associated with activated cytolytic natural killer cells expressing high levels of T cell immunoglobulin-and mucin-domain-containing molecule-3. Clin Immunol. 2015;158:114-125.

29. Li M, Ablan SD, Miao C, Zheng YM, Fuller MSTIM-family proteins inhibit HIV-1 release. Proc Natl Acad Sci USA. 2014;111:E3699-E3707.

30. Cai C, Xu YF, Wu ZJ, Dong Q, Li MY, Olson JC, Rabinowitz YM, Wang LH, Sun YH. TIM3 expression represents dysfunctional tumor infiltrating T cells in renal cell carcinoma. World J Urol. 2016; 
34(4):561-7.

31. Jinpeng Z, Yang J, Haiying Z, Lian C, Peng L, Long L, Zhao JS, Lv F, Zou D, Zhang Y, Jing ZT. Clinicopathological implications of TIM3+ tumor-infltrating lymphocytes and the miR-455-5p/Galectin-9 axis in skull base chordoma patients Cancer Immunology. Immunotherapy. 2019; 68:1157-1169.

32. Jie HB, Srivastava RM, Argiris A, Bauman JE, Kane LP, Ferris RL. Increased PD-1 + and TIM3 + TILs during Cetuximab Therapy Inversely Correlate with Response in Head and Neck Cancer Patients. Cancer Immunol Res.2017;5(5):408-416.

33. Shariati S, Ghods A, Zohouri M, Rasolmali R, Talei AR, Mehdipour F, Ghaderi A. Significance of TIM3 expression by $\mathrm{CD} 4+$ and $\mathrm{CD} 8+\mathrm{T}$ lymphocytes in tumor-draining lymph nodes from patients with breast cancer. Mol Immunol. 2020; 128:47-54.

34. Ngiow SF, von Scheidt B, Akiba H, Yagita H, Teng MW, Smyth MJ. Anti-TIM3 Antibody Promotes T Cell IFN-YMediated Antitumor Immunity and Suppresses Established Tumors. Cancer Res. 2011;71:3540-3551.

35. Du W, Yang M, Turner A, Xu C, Ferris RL, Huang J, Lawrence P, Binfeng Lu BF. TIM3 as a Target for Cancer Immunotherapy and Mechanisms of Action.Int J Mol Sci. 2017;18(3):645.

36. Acharya N, Sabatos-Peyton C, Anderson AC. TIM3 finds its place in the cancer immunotherapy landscape. Journal for Immunother Cancer. 2020;8:e000911.

37. He Y, Cao J, Zhao C, Li X, Zhou C, Hirsch FR. TIM3, a promising target for cancer immunotherapy.OncoTargets and Ther. 2018;11:7005-7009.

38. Yang R, Cheng S, Luo N, Gao R, Yu K, Kang B, Wang L, Zhang QM, Fang Q, Zhang L, Li C, He A, Hu X, Peng J, Ren XW, Z Zhang Z. Distinct epigenetic features of tumorreactive CD8+ T cells in colorectal cancer patients revealed by genome-wide DNA methylation analysis. Genome Biol.2020; 21:2.

39. Seo H, Chen J, González-Avalos E, Samaniego-Castruita D, Das A, Wang YH, López-Moyado IF, Georges RO, Zhang W, Onodera A, Wu CJ, Lu LF, Hogan PG, Bhandoola A, Rao A. TOX and TOX2 transcription factors cooperate with NR4A transcription factors to impose CD8(+) T cell exhaustion. Proc Natl Acad Sci USA. 2019; 116(25):12410-12415.

40. Quigley M, Pereyra F, Nilsson B, Porichis F, Fonseca C, Eichbaum Q, Julg B, Jesneck JL, Brosnahan K, Imam S, Russell K, Toth I, Piechocka-Trocha A, Dolfi D, Angelosanto J, Crawford A, Shin H, Kwon DS, Zupkosky J, Francisco L, Freeman GJ, Wherry EJ, Kaufmann DE, Walker BD, Ebert B, Haining WN. Transcriptional analysis of HIV-specific CD8+ T cells shows that PD-1 inhibits T cell function by upregulating BATF. Nat Med. 2010;16(10):1147-51.

41. Chen J, López-Moyado IF, Seo H, Lio CJ, Hempleman LJ, Sekiya T, Sekiya T, Yoshimura A, JScottBrowne JP, Rao A. Nr4a transcription factors limit CAR T cell function in solid tumors. Nature. 2019; 567(7749): 530-534.

42. Zolov SN, Rietberg SP, Bonifant CL. Programmed cell death protein 1 activation preferentially inhibits CD28CAR-T cells. Cytotherapy. 2018;20(10):1259-1266. 
43. Funk CR, Petersen CT, Jagirdar N, Ravindranathan S, Jaye DL, Flowers CR, Langston A, Waller EK.Oligoclonal T Cells Transiently Expand and Express TIM3 and PD-1 Following Anti-CD19 CAR T Cell Therapy: A Case Report.Int J Mol Sci. 2018;19(12):4118

44. Liang X, Gu J, Li TJ, Zhao L, Fu X, Zhang W, Wang J, Shang Z, Huang W, Zhou JF. PAX5 haploinsufficiency induce cancer cell dormancy in Raji cells. Exp Cell Res,2018 Jun 1;367(1):30-36.

Figures 


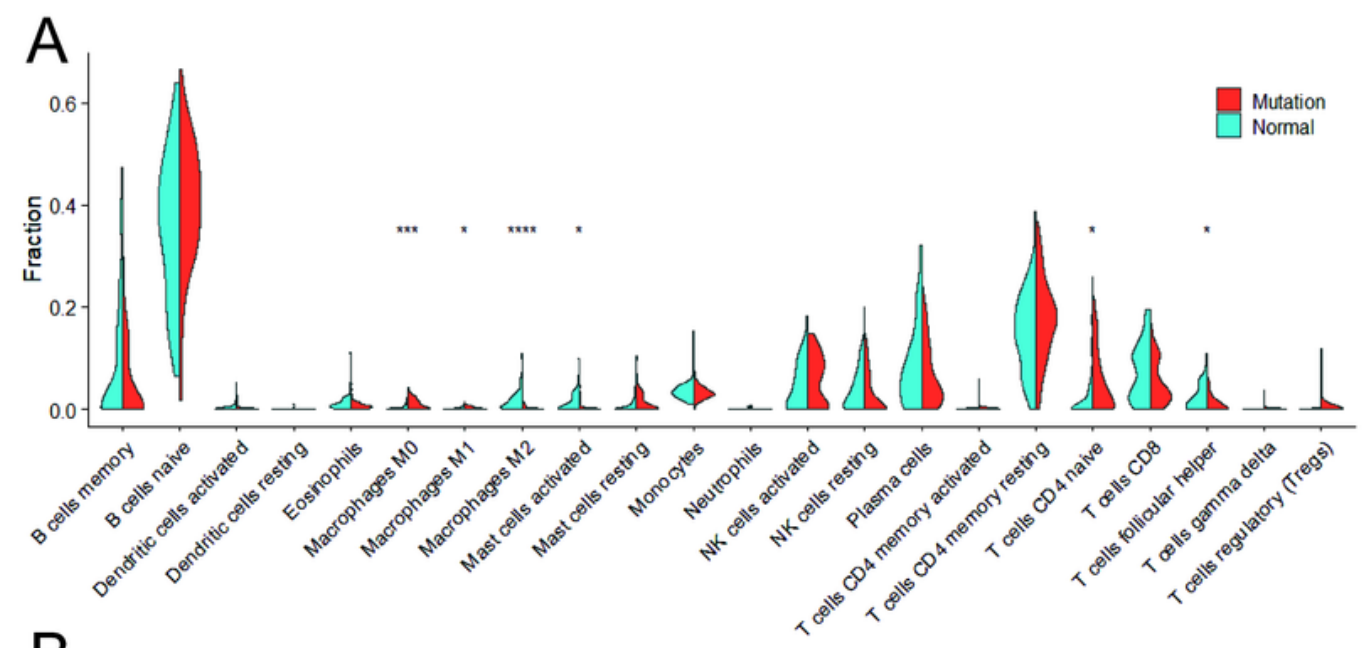

$\mathrm{B}$
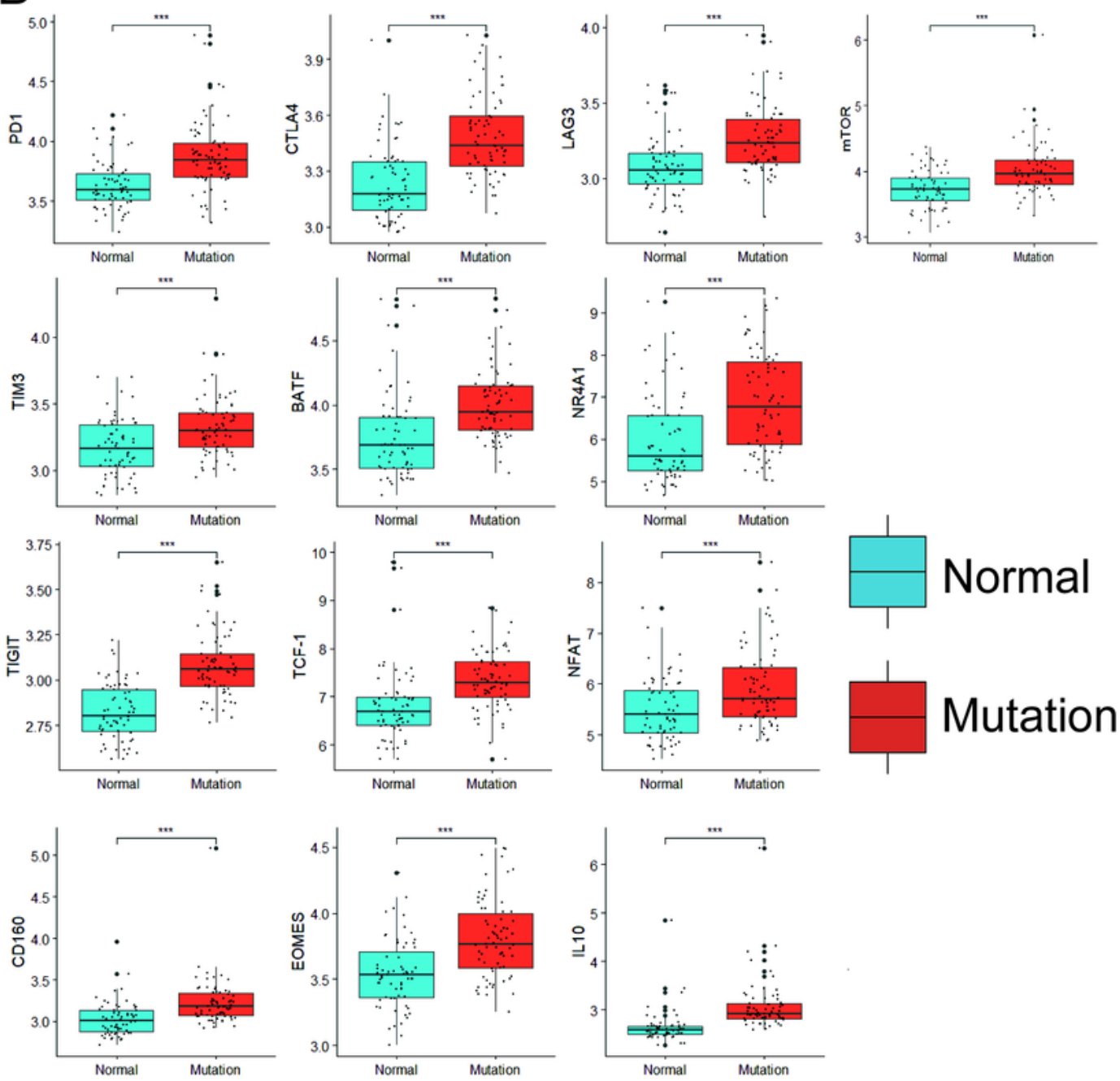

Figure 1

The compositions of immune cell subsets and the expressions of immune inhibitory-related molecules in bone marrow of B-ALL. (A) The violin plot showed the percentage difference of immune cell subsets between B-ALL with PAX5 mutations $(n=70)$ and B-ALL without PAX5 mutations $(n=64)$. (B) The expression difference of immune inhibitory-related molecules between B-ALL with PAX5 mutations $(n=70)$ and B-ALL without PAX5 mutations $(n=64)$. * means $p<0.05$; *** means $p<0.001$; **** means $p<0.0001$. 
A

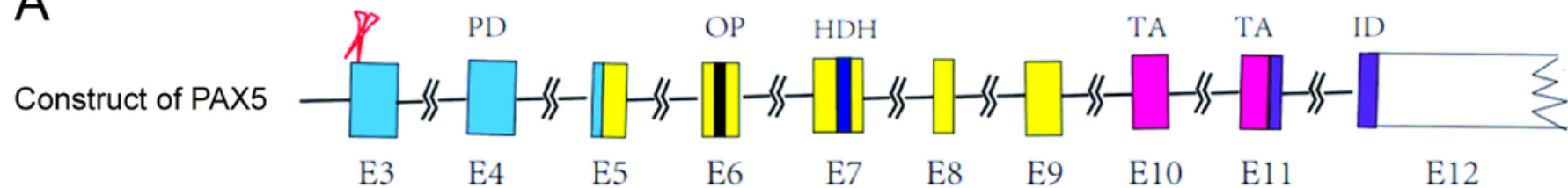

WT

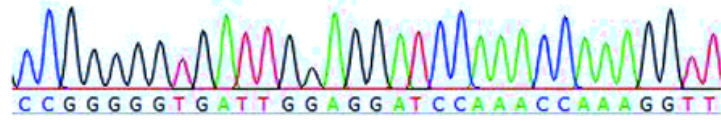

WT CCGGGGGTGATTGGAGGATCCAAACCAAAGGTT

MUT1 CCGGGGGTGAT ${ }^{* *}$ GAGGATCCAAACCAAAGGTT

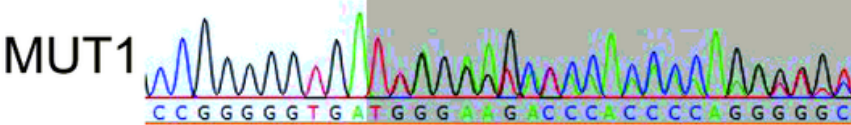

WT CCGGGGGTGATTGGAGGATCCAAACCAAAGGTT

MUT2 NWWWNAwawh Nh NhA

MUT2 CCGGGGGTGATTTGAGGATCCAAACCAAAGGTT

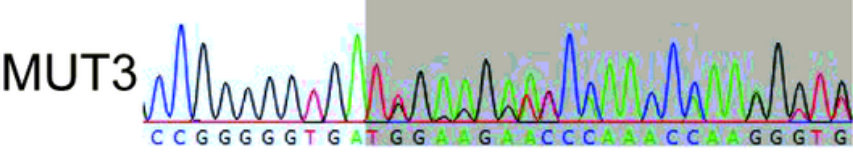

WT CCGGGGGTGATTGGAGGATCCAAACCAAAGGTT

MUT3 CCGGGGGTGAT*GGAGGATCCAAACCAAAGGTT

B

C

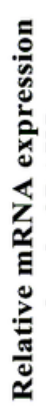

党
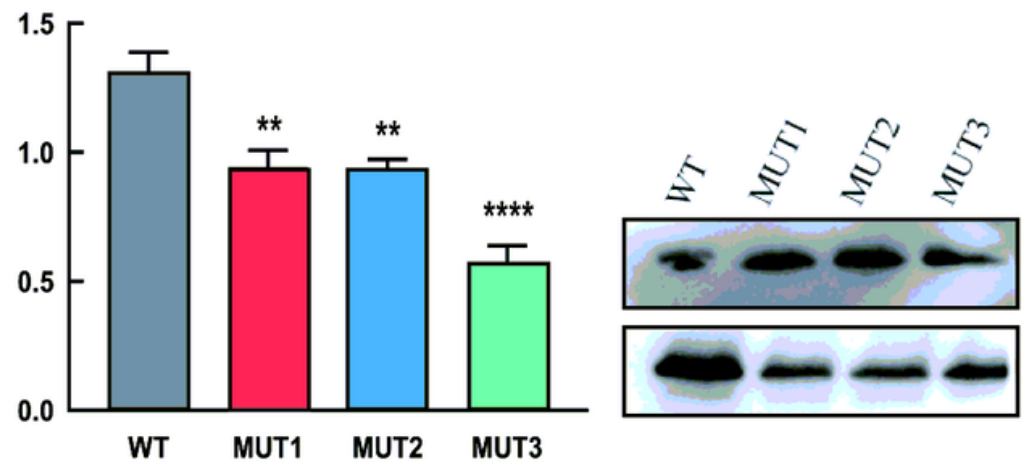

$\beta$-actin

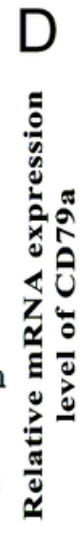

Figure 2

Identification of PAX5 haplodeletion in A20 cells. A: The construction of PAX5 and haplodeletion type of PAX5. E3 was the target of gene editing operation, and sequencing analyses of edited E3 regions. B: The mRNA level of PAX5 tested by quantitative RT-PCR(n=3). C: Protein level of PAX5 tested by western blot( $(n=3)$. D: Downstream target genes tested by quantitative RT-PCR(n=3). E: exon of PAX5; PD: paired box domain; OP: octapeptide domain; HD: homeodomain; TA: transactivation domain; ID: inhibitory domain; WT: wild-type control; MUT: mutation clone. The data are expressed as the mean $\pm S D$ and

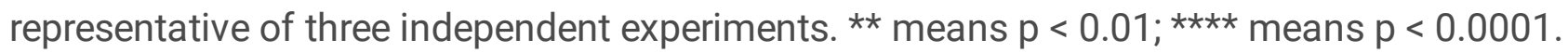



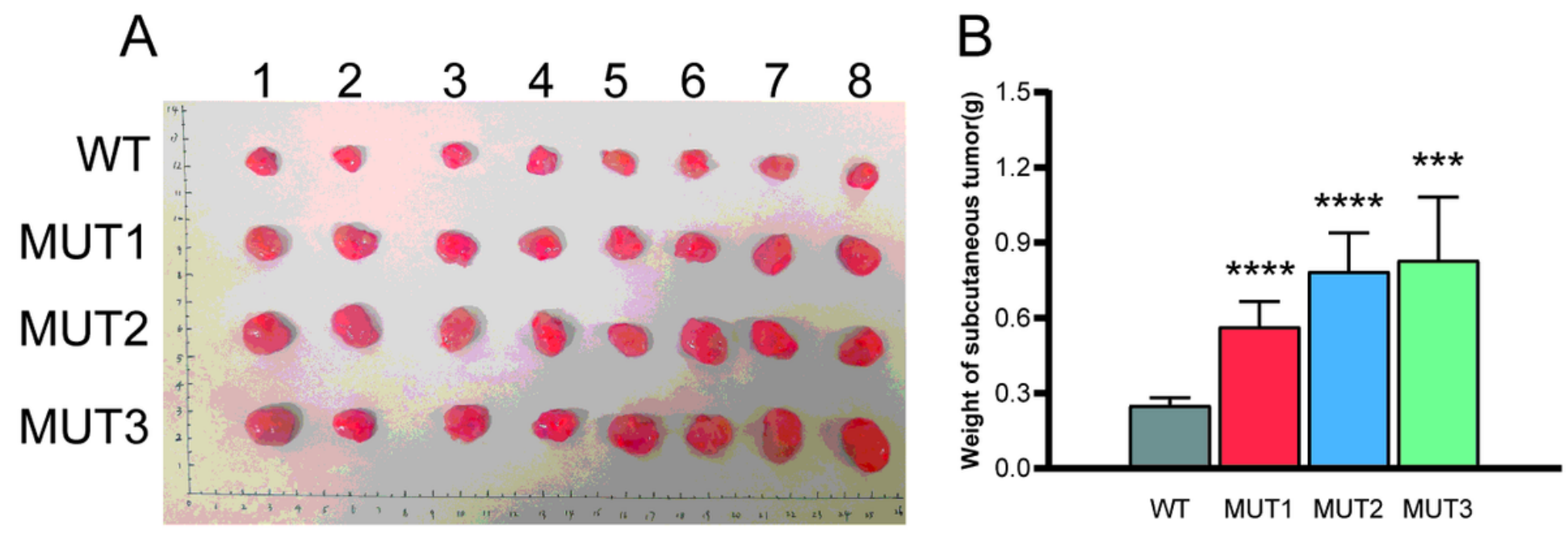

Figure 3

PAX5 haplodeletion influenced tumor formation in BALB/c mice $(n=10)$. The data are expressed as the

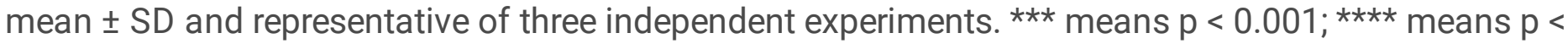
0.0001 . 

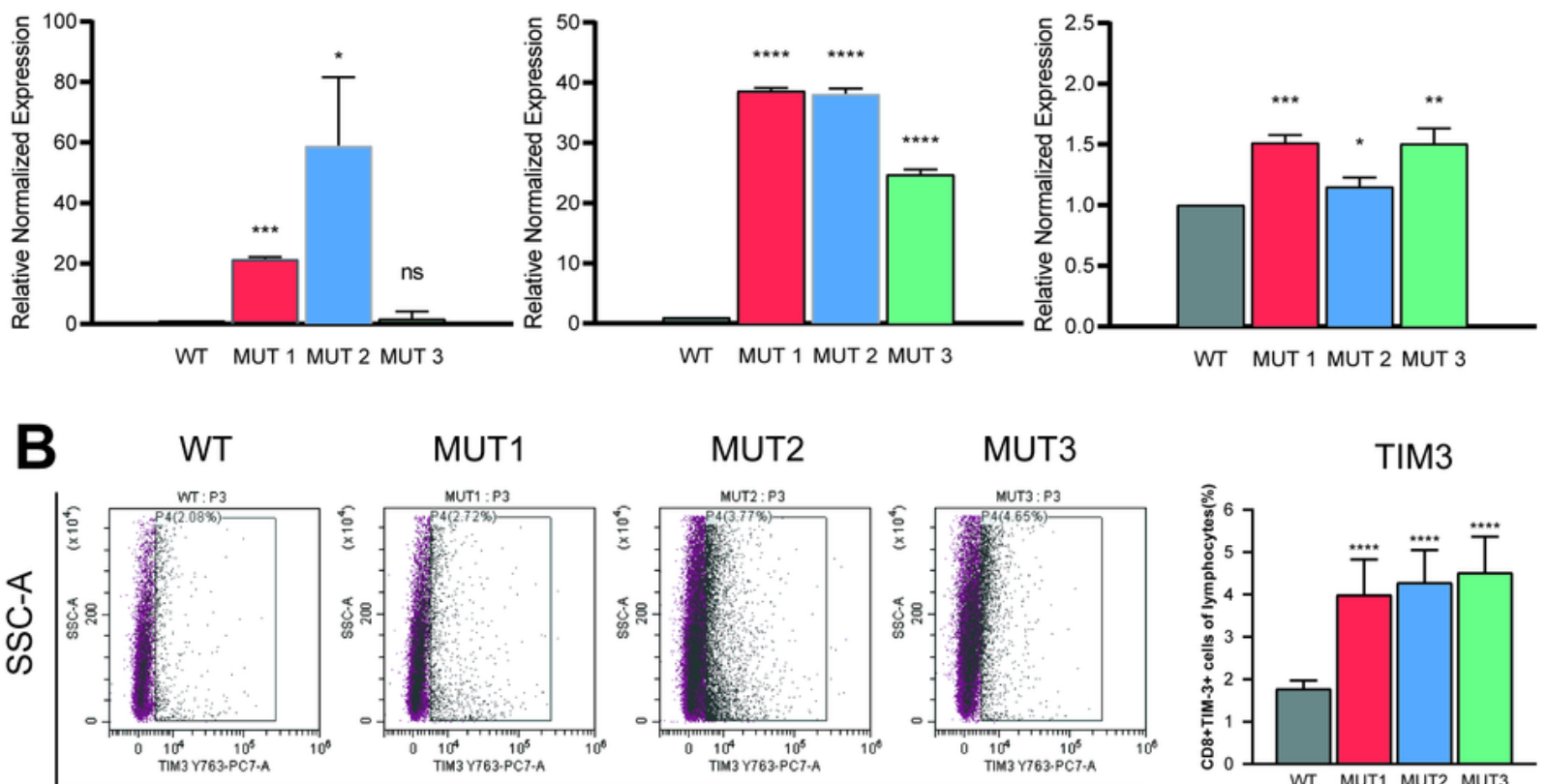

MUT1

MUT2

MUT3
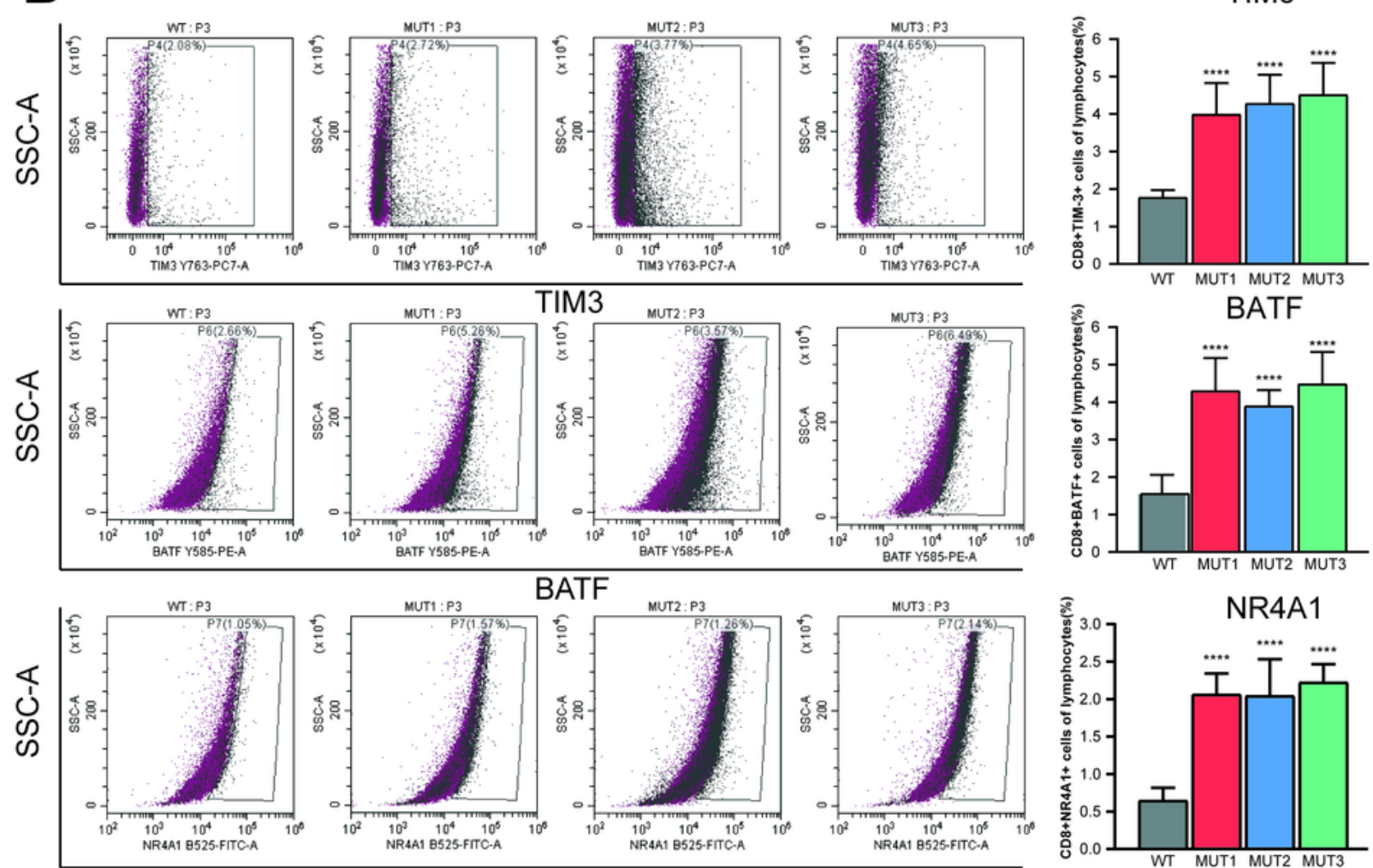

NR4A1

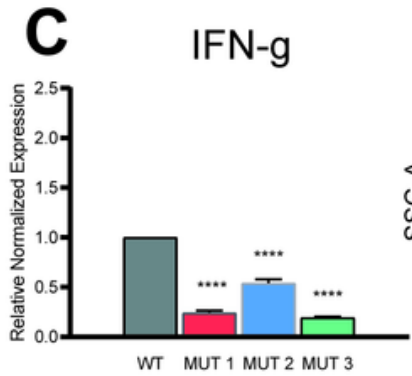

WT

MUT1

MUT2

MUT3

IFN-g
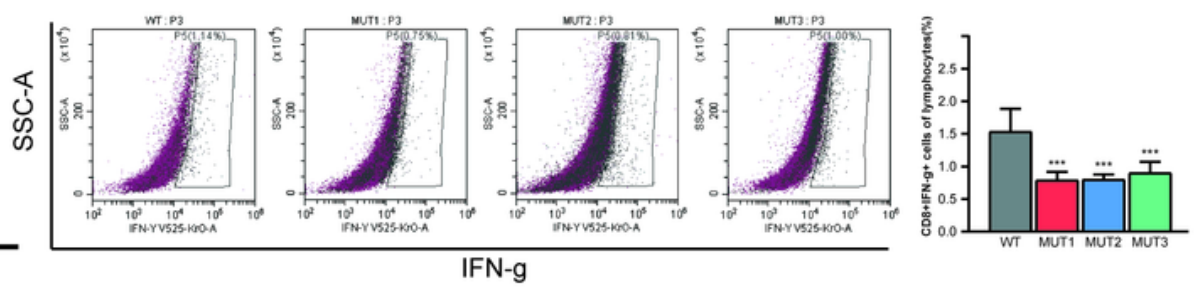

Figure 4

PAX5 haplodeletion influenced expression of immune inhibitory-related molecules and INF- $\mathrm{X}$. A.The mRNA level of TIM3, NR4A1 and BATF of sorted CD8+ T cells in PAX5 haplodeletion tumors were significantly higher than that in wild type control tested by quantitative RT-PCR $(n=3)$. B: The protein level of TIM3, NR4A1 and BATF of CD8+ T cells in PAX5 haplodeletion tumors were significantly higher than that in wild type control tested by by Flow cytometry $(n=6)$. C: The mRNA and protein level of INF-g of CD8+ T cells 
was significantly lower in PAX5 haplodeletion clones than that in wild-type control( $n=3$ and $n=6$, respectively). The data are expressed as the mean $\pm S D$ and representative of three independent

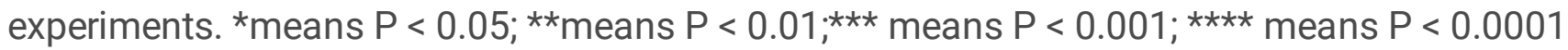

\section{Supplementary Files}

This is a list of supplementary files associated with this preprint. Click to download.

- CD79APCR.png

- CD79a.dat

- PAX5PCR.png

- SupplementaryAppendixTable1.docx

- SupplementaryAppendixTable2.docx

- pax5western.tif 